\title{
Renormalization Group approach to Inhomogeneous Cosmology
}

\author{
J. Ibáñez and S. Jhingan \\ Departamento de Física Teórica, Universidad del País Vasco, Apartado 644, 48080, Bilbao, Spain
}

(Dated: March 6, 2018)

\begin{abstract}
Soliton solutions are recovered as scale-invariant asymptotic states of vacuum inhomogeneous cosmologies using renormalization group method. The stability analysis of these states is also given.

PACS numbers: 98.80.Jk, 64.60.Ak
\end{abstract}

\section{INTRODUCTION}

The study of influence of initial inhomogeneities upon the evolution of cosmological models is an important issue in Cosmology, both in order to understand the formation of large-scale structures as well the smoothing away of these inhomogeneities. One way to deal with the inhomogeneities is to consider perturbations of known solutions, like Friedmann-Robertson-Walker models or homogeneous Bianchi type solutions. Another way is to assume inhomogeneous solutions from the beginning and study their dynamical evolution; it is this that we are concerned with here. The simplest inhomogeneous models are the so called diagonal $G_{2}$ cosmologies for which the spacetime admits two commuting Killing vectors whose orbits are 2-dimensional space-like surfaces. They represent inhomogeneities of spatially homogeneous models and can be considered as gravitational waves of a single polarization propagating over a homogeneous background [1]. Using a few solution generating techniques a large number of exact solutions with different sources have been found [2].

The asymptotic evolution of homogeneous Bianchi type models and both their behavior near the initial singularity as well as their future state have been widely studied [3]. One of the facts relevant to our paper is that at late times Bianchi models can be described as self-similar solutions. However, the asymptotic behavior of inhomogeneous solutions, particularly $G_{2}$ metrics, revealed a task much more difficult than that of homogeneous models. Contrary to the homogeneous metrics for which the Einstein equation reduce to an autonomous system of differential equations that can be analyzed using techniques from the theory of dynamical systems, the field equations of inhomogeneous spacetimes are partial differential equations. In ref. [3] the evolution of a particular class of $G_{2}$ metrics is studied showing that in that case all the solutions are asymptotically self-similar. The same result was obtained in [4] and in [5] where special families of $G_{2}$ solutions were considered with a scalar field. On the other side, it has been suggested that fluctuations might evolve from arbitrary initial conditions to self-similar form [ [] . From all these results it is reasonable to regard the self-similar solutions as describing the long time asymptotic of inhomogeneous metrics. Hence it would be worthy to analyze the asymptotic behavior of general class of $G_{2}$ metrics by using a method that emphasizes the scaling properties of the underlying field equations.

In this paper we study the asymptotic evolution of vacuum $G_{2}$ metrics using a different approach than that used in the above referred papers: we make use of renormalization group (RG) tools to study the structurally stable characteristics of the inhomogeneous metrics. Recently, RG techniques have been exhibited as a powerful implement to study the asymptotic behavior of partial differential equations [7, 8, 9, 10]. The RG method has been applied to study homogeneous and isotropic universe, an spherically symmetric dust collapse [1], critical phenomena related with gravitational collapse [12], Newtonian cosmology [13], homogeneous flat causal bulk viscous cosmological models [14] and the theory of perturbations of isotropic universe with dynamically evolving Newton constant and cosmological constant [15, 16].

The plan of the paper is the following: in Section 1 we illustrate the application of the RG method to a homogeneous Bianchi type metric with a scalar field. This case has been studied before and we recover the attractors of the system by means of the RG method. In Section $\amalg$ we apply the RG technique to the vacuum, diagonal, $G_{2}$ metric. We find the fixed-points and analyze their stability as well. We conclude with Section IV

\section{THE RG METHOD: AN ILLUSTRATIVE EXAMPLE}

It is well known that the asymptotics of partial differential equations can often be found from the consideration of scaling solutions (the equivalence of RG theory and the theory of intermediate asymptotics was shown by Goldenfeld et. al. [17]). Though it is usual practice to find the similarity variable to analyze scaling solutions from a combination of variables using dimensional arguments, however, there is a large class of problems where this cannot be done [18]. $\mathrm{RG}$ provides a systematic approach for finding the scaling variables as well as the asymptotics of partial differential equations. 
The general (RG) method adapted to partial differential equations is well known 7]. Therefore, instead of reproducing the whole procedure, we explain this method using an illustrative example which has been studied with other methods and has a non-trivial asymptotic structure. We consider a class of anisotropic cosmological models given by 19$]$

$$
d s^{2}=-d t^{2}+a(t)^{2} d x^{2}+b(t)^{2} e^{2 m x} d y^{2}+c(t)^{2} e^{2 x} d z^{2}
$$

This represents a one parameter $(m)$ family of Bianchi models with Bianchi III, $\mathrm{V}$ and $\mathrm{VI}_{0}$ for, respectively, $m=0,1$ and -1 , and Bianchi $\mathrm{VI}_{\mathrm{h}}(h=m+1)$ for all other vlaues.

For a direct comparison with earlier works we choose the same variables, namely, shear $(\sigma)$ and expansion $(\theta)$ for our analysis. Note, however, that the feasibility of the earlier analysis depends crucially on finding the "right" set of variables to work-with, otherwise system is too complex to analyze completely, even in this homogeneous case. Moreover, the choice of these variables is model dependent and rather ad-hoc. Therefore, one also needs to be lucky to be able to analyze. Whereas, with RG one can directly work with the metric functions, as will be done in the next Section.

The shear and expansion for this spacetime are given by

$$
\begin{aligned}
\theta & =\frac{\dot{a}}{a}+\frac{\dot{b}}{b}+\frac{\dot{c}}{c} \\
\sigma^{2} & =\frac{1}{3}\left[\left(\frac{\dot{a}}{a}\right)^{2}+\left(\frac{\dot{b}}{b}\right)^{2}+\left(\frac{\dot{c}}{c}\right)^{2}\right]
\end{aligned}
$$

Here an over-dot signifies derivative with respect to $t$. We consider energy momentum tensor describing a minimally coupled homogeneous scalar field $\phi(t)$, given by

$$
T_{\mu \nu}=\phi_{, \mu} \phi_{, \nu}-g_{\mu \nu}\left(\frac{1}{2} \phi_{, \alpha} \phi^{, \alpha}+V(\phi)\right),
$$

with an exponential potential $V(\phi)=\lambda \exp (k \phi)$. Here, $\lambda>0$ and $k$ are both constants. The evolution equations are then given by

$$
\begin{aligned}
\dot{\theta} & =-2 \sigma^{2}-\frac{\theta^{2}}{3}-\dot{\phi}^{2}+V(\phi) \\
\dot{\sigma} & =-\sigma \theta+p(m)\left(\theta^{2}-3 \sigma^{2}-\frac{3}{2} \dot{\phi}^{2}-3 V(\phi)\right), \\
\ddot{\phi} & =-\theta \dot{\phi}-k V(\phi) \\
\dot{V} & =k \dot{\phi} V,
\end{aligned}
$$

where $p(m)=(1-m) /\left(3 \sqrt{3\left(1+m+m^{2}\right)}\right)$. Since above system is an autonomous system of differential equations, in the previous study [19] the theory of dynamical systems was used to analyze its asymptotic behavior using "expansionnormalized variables". We will use, as an alternative method, the RG technique and recover the same results. Again, our goal is to illustrate the method in this section and is applied it to a new scenario in next section.

We find it useful to work with a compact notation and, therefore, define a new indexed variable $u_{i}(t)$ that signifies the set $\{\theta, \sigma, \dot{\phi}, V\}$, for $i=1,2,3,4$, respectively. Our interest is in the asymptotics of solution of the form

$$
\lim _{t \rightarrow \infty} u_{i}(t)=t^{-\alpha_{i}} u_{i}^{*}(1)
$$

where argument "1" signifies initial value of the quantity. It is convenient to fix initial time as $t=1$. Now we will illustrate how RG method gives a systematic procedure to fix $\alpha_{i}$ and determine the scaling function $u_{i}^{*}(1)$ as fixed-points of RG equations (in a inhomogeneous case the argument of $u_{i}^{*}$ will not be a constant but will depend on a combination of time and spatial variable, the scaling variable).

Let us consider transformations of the form

$$
\begin{aligned}
t & \longrightarrow L t \\
u_{i}(t) & \longrightarrow U_{i}(t)=L^{\alpha_{i}} u_{i}(L t),
\end{aligned}
$$


which leave the equations invariant, i.e., if $u_{i}(t)$ is a solution so is $U_{i}(t)$. We use a number $L>1$ as a parameter of scale transformation. Note that unlike in the application of RG method to quantum field theories or statistical mechanics, there is no natural way to choose a scale $L$ here. Moreover, $L$ never appears explicitly in the equations.

From equation set (2.5)-2.8) and (2.10) it is straightforward to recover

$$
\alpha_{1}=\alpha_{2}=\alpha_{3}=\frac{\alpha_{4}}{2}=1
$$

Now, defining $L=\exp (\tau)$, and using Eq. 2.10) in (2.5) (2.8) we get a new set of evolution equations:

$$
\frac{d U_{i}}{d \tau}=\alpha_{i} U_{i}+\frac{\partial U_{i}}{\partial t}
$$

The procedure of defining $\tau$ is, in a sense, analogous to summing over all degrees of freedom corresponding to fluctuations of scale less than $L$ and then re-scaling everything by $L^{-1}$. This new set of evolution equations define the RG transformations. Since scaled quantities satisfy same evolution equations the time derivative in the equation set above can be replaced with equations (2.5)-(2.8), and we have

$$
\begin{aligned}
& \frac{d U_{1}}{d \tau}=U_{1}-2 U_{2}^{2}-\frac{U_{1}^{2}}{3}-U_{3}^{2}+U_{4} \\
& \frac{d U_{2}}{d \tau}=U_{2}-U_{1} U_{2}+p(m)\left(U_{1}^{2}-3 U_{2}^{2}-\frac{3}{2} U_{3}^{2}-3 U_{4}\right), \\
& \frac{d U_{3}}{d \tau}=\left(1-U_{1}\right) U_{3}-k U_{4}, \\
& \frac{d U_{4}}{d \tau}=\left(2+k U_{3}\right) U_{4} .
\end{aligned}
$$

All the quantities on the right hand side are evaluated at $t=1$. Scale-invariant solutions emerge now from the fixed-point structure of the RG map, which is defined by

$$
\frac{d U_{i}^{*}}{d \tau}=0
$$

with $U_{i}{ }^{*}$ being the fixed-points. The complete set of fixed-points for this system are given in the table below :

\begin{tabular}{|c|c|c|c|c|}
\hline & $U_{1}$ & $U_{2}$ & $U_{3}$ & $U_{4}$ \\
\hline 1. & 1 & $\pm \sqrt{\frac{2-3 U_{3}}{6}}$ & $U_{3}$ & 0, \\
\hline 2. & $\frac{6}{k^{2}}$ & 0 & $-\frac{2}{k}$ & $2 \frac{\left(6-k^{2}\right)}{k^{4}}$ \\
\hline 3. & $\frac{2(1-m)^{2}}{27 \mathrm{p}(\mathrm{m})^{2}\left(1+m^{2}\right)}$ & $\frac{(1-m)^{2}}{9 \mathrm{p}(\mathrm{m})\left(1+m^{2}\right)}$ & 0 & 0 \\
\hline 4. & $\left(1+\frac{k^{2}}{27 \mathrm{p}(\mathrm{m})^{2}}\right) \frac{2(1-m)^{2}}{k^{2}\left(1+m^{2}\right)}$ & $\frac{\left(k^{2}-2\right)}{9 k^{2} \mathrm{p}(\mathrm{m})} \frac{(1-m)^{2}}{\left(1+m^{2}\right)}$ & $-\frac{2}{k}$ & $\frac{2(1+m)^{2}}{k^{2}\left(1+m^{2}\right)}+\frac{4(1-m)^{2}}{k^{4}\left(1+m^{2}\right)}$ \\
\hline
\end{tabular}

Table I: Fixed-points of Bianchi $V I_{h}$ models

Therefore, we have four exact scale invariant solutions of the form (2.9) with exponents $\alpha_{i}$ given by (2.11) and $u_{i}^{*}(1)$ being the fixed-points given above. Since the stability properties of these solutions is well studied in literature [19], we move now to the more general inhomogeneous case. We analyze the fixed point structure as well as their stability properties, which is not done before.

\section{INHOMOGENEOUS CASE}

We begin with a family of metrics which have been a very useful tool for studying inhomogeneous cosmological models, known as the generalized Einstein-Rosen spacetimes (see for example, [20, 21]). The line element is of the 
form:

$$
d s^{2}=f^{2}\left(d z^{2}-d t^{2}\right)+\gamma_{a b} d x^{a} d x^{b}, \quad a, b=1,2 .
$$

Here $x^{1}=x, x^{2}=y$, and both $f$ and $\gamma_{a b}$ are functions only of $z$ and $t$. Metric (3.1) is fairly general and includes Bianchi type models I to VII. In this paper we are interested in vacuum solutions only. We can impose now

$$
\operatorname{det} \gamma_{a b}=t^{2},
$$

since in vacuum case one of the field equation takes the form

$$
\left(\operatorname{det} \gamma_{a b}\right)_{, t t}^{(1 / 2)}-\left(\operatorname{det} \gamma_{a b}\right)_{, z z}^{(1 / 2)}=0 .
$$

$t$ and $z$ are two independent solutions of this equation. Under this condition the coordinates $t$ and $z$ are called as the canonical coordinates; and there is no loss of generality in this choice 20]. To simplify the analysis we now specialize to the diagonal metrics, i.e., metrics with a single polarization.

The spacetime can be written in the form

$$
d s^{2}=f^{2}\left(d z^{2}-d t^{2}\right)+t\left(h^{2} d x^{2}+h^{-2} d y^{2}\right),
$$

where $f$ and $h$ are functions of $t$ and $z$ only. These metrics admit an Abelian $G_{2}$ group of isometries with 2 spacelike commuting killing vectors $\partial_{x}$ and $\partial_{y}$. The set of Einstein field equations in the vacuum case reduces to

$$
\begin{aligned}
h_{, t} & =\frac{1}{2 t} \frac{f_{, z}}{f} \frac{h^{2}}{h_{, z}} \\
f_{, t} & =-\frac{1}{4 t} f+t f\left(\frac{h_{, z}}{h}\right)^{2}+\frac{1}{4 t} \frac{\left(f_{, z}\right)^{2}}{f}\left(\frac{h}{h_{, z}}\right)^{2} .
\end{aligned}
$$

Eqs. (3.5) and (3.6) are the evolution equations for $h$ and $f$, respectively, and it is easy to check that the remaining field equations are identically satisfied. We now follow the prescription given in the previous section to recover the exact scaling solutions.

Let us consider the following scale transformation

$$
\begin{aligned}
z & \longrightarrow L z \\
t & \longrightarrow L^{\epsilon} t \\
h(t, z) & \longrightarrow \phi(t, z)=L^{\alpha} h\left(L^{\epsilon} t, L z\right), \\
f(t, z) & \longrightarrow \psi(t, z)=L^{\beta} f\left(L^{\epsilon} t, L z\right) .
\end{aligned}
$$

Here $\phi$ and $\psi$ are the scaled quantities. Since scaled quantities also satisfy the original equations, Eq. (3.6) fixes

$$
\epsilon=1 \text {. }
$$

The scaling relations (3.7) along with (3.8) and successive transformations, first $t \rightarrow 1$ and then $L \rightarrow t$, gives scale invariant solution of the form

$$
\begin{aligned}
& h(t, z)=t^{-\alpha} \phi(1, z / t), \\
& f(t, z)=t^{-\beta} \psi(1, z / t) .
\end{aligned}
$$

The equations above express arbitrary solution in terms of initial data (at $t=1$ ). As stated earlier we work with $t=1$ as our initial time and evolution is in sense of scaled time $L t$ with $L>1$.

From simple dimensional analysis we would have $\beta=0$ and $\alpha= \pm 1 / 2$ (introducing a dimensional constant multiplying either $d x$ or $d y$ ). However, as we will see later this would lead to a trivial solution: the homogeneous Kasner metric. The fact that we recover Kasner solution as a fixed point of a general inhomogeneous G2 metric though is non-trivial and it elucidates the appearance of this solution in earlier studies; it is used as a seed metric in different solution generating techniques, and more importantly, is known to describe "generic" cosmological singularity in the analysis of Belinskii et al. [22]. In order to get a non-trivial structure in fix-point analysis we need to analyze the anomalous dimensions for both the functions $f$ and $h$. It is well known $[23$ that the anomalous dimensions $\alpha \neq \pm 1 / 2$ and $\beta \neq 0$ are fixed by initial or boundary conditions. In our case, cosmological vacuum solutions, initial condition refers to geometry at $t=0$. Since, metrics of the type (3.4) are singular at $t=0$ there is not strict functional 
constraint on the system described by (3.5) and (3.6). Nevertheless, interpreting a posteriori the fixed points we can give some hints to understand the role of anomalous dimensions $\alpha$ and $\beta$.

Denoting $L=\exp (\tau)$ the RG equations are

$$
\begin{aligned}
& \frac{d \phi}{d \tau}=\alpha \phi+\phi^{\prime} z+\frac{1}{2}\left(\frac{\psi^{\prime}}{\psi}\right)\left(\frac{\phi^{2}}{\phi^{\prime}}\right), \\
& \frac{d \psi}{d \tau}=\left(\beta-\frac{1}{4}\right) \psi+\psi^{\prime} z+\psi\left(\frac{\phi^{\prime}}{\phi}\right)^{2}+\frac{1}{4} \frac{\left(\psi^{\prime}\right)^{2}}{\psi}\left(\frac{\phi}{\phi^{\prime}}\right)^{2} .
\end{aligned}
$$

We now investigate the fix-point structure of the equation set above, i.e.,

$$
\begin{aligned}
& \frac{d \phi^{*}}{d \tau}=0 \Rightarrow \alpha=-\left(\frac{\phi^{*^{\prime}}}{\phi^{*}}\right) z-\frac{1}{2}\left(\frac{\psi^{*^{\prime}}}{\psi^{*}} \frac{\phi^{*}}{\phi^{*^{\prime}}}\right), \\
& \frac{d \psi^{*}}{d \tau}=0 \Rightarrow \frac{1}{4}-\beta=\left(\frac{\psi^{*^{\prime}}}{\psi^{*}}\right) z+\left(\frac{\phi^{*^{\prime}}}{\phi^{*}}\right)^{2}+\frac{1}{2}\left(\frac{\psi^{*^{\prime}}}{\psi^{*}} \frac{\phi^{*}}{\phi^{*^{\prime}}}\right)^{2} .
\end{aligned}
$$

System decouples to give

$$
\begin{aligned}
& \left(\frac{\phi^{*^{\prime}}}{\phi^{*}}\right)= \pm \sqrt{\frac{\triangle}{z^{2}-1}} \\
& \left(\frac{\psi^{*^{\prime}}}{\psi^{*}}\right)=\frac{2 \sqrt{\triangle}}{1-z^{2}}\left(\sqrt{\triangle} z \pm \alpha \sqrt{z^{2}-1}\right),
\end{aligned}
$$

where $\triangle=\alpha^{2}+\beta-1 / 4$. The real solutions correspond to $\Delta>0$ for $z^{2}>1$ and $\triangle<0$ for $z^{2}<1$. The equations (3.14) and (3.14) can be easily integrated to give the fixed-points

$$
\begin{aligned}
& \phi^{*}=\left(z+{\sqrt{z^{2}-1}}^{ \pm \sqrt{\Delta}},\right. \\
& \psi^{*}=c_{f}\left(z+\sqrt{z^{2}-1}\right)^{\mp 2 \alpha \sqrt{\Delta}}\left(z^{2}-1\right)^{-\triangle},
\end{aligned}
$$

were $c_{f}$ is an integration constant. We have dropped constant of integration from $\psi^{*}$ since this can be absorbed simply by scaling of $x$ and $y$. Also, we note here that the new parameter $\triangle$ relates our spacetime metric with Kasner metric in the following way. The Kasner metric can be written in the form

$$
d s^{2}=t^{\left(d^{2}-1\right) / 2}\left(d z^{2}-d t^{2}\right)+t^{1+d} d x^{2}+t^{1-d} d y^{2},
$$

where parameter $d$ can be chosen positive or negative. A direct comparison with the metric (3.4) using (3.9) gives the Kasner relationship

$$
4 \alpha^{2}+4 \beta-1=0 .
$$

Therefore, $\triangle=0$ case corresponds to the Kasner models. When $\triangle>0\left(z^{2}>t^{2}\right)$ the critical solution is:

$$
\begin{aligned}
& h(t, z)=t^{-\alpha \mp \sqrt{\triangle}}\left(z+\sqrt{z^{2}-t^{2}}\right)^{\sqrt{\Delta}}, \\
& f(t, z)=c_{f} t^{(\alpha \pm \sqrt{\Delta}+1 / 2)(\alpha \pm \sqrt{\Delta}-1 / 2)}\left(z+\sqrt{z^{2}-t^{2}}\right)^{\mp 2 \alpha \sqrt{\triangle}}\left(z^{2}-t^{2}\right)^{-\triangle} .
\end{aligned}
$$

and when $\triangle<0\left(z^{2}<t^{2}\right)$ the critical solution is:

$$
\begin{aligned}
& h(t, z)=t^{-\alpha} \exp \left(\mp \sqrt{-\triangle} \arccos \frac{z}{t}\right) \\
& f(t, z)=c_{f}^{\prime} t^{\alpha^{2}+\triangle-1 / 4}\left(t^{2}-z^{2}\right)^{-\triangle} \exp \left( \pm 2 \alpha \sqrt{-\triangle} \arccos \frac{z}{t}\right) .
\end{aligned}
$$

The spacetime is split into two regions separated by the light-cone $z=t$. In each region the solution takes one of the forms given by the expressions above. For $\Delta=0$ we have the Kasner metric. In the general case $(\Delta \neq 0)$, solution (3.19) is the soliton metric that has been obtained earlier by the inverse scattering transformation with real poles from the Kasner metric, and the solution (3.20) is the cosoliton solution generated also from the Kasner metric. 
Both solutions have been studied in [24] (and references therein). Depending of the parameters, the light-cone $z=t$ is singular for the solution (3.19) but is always singular for (3.20). This means that even though the metric and its first derivatives are continuous across the light-cone, the solutions cannot be matched across the light-cone (for a discussion on the matching of these metrics see [24]). We would like to note here that the \pm sign in the above equations is actually related to the symmetry under $x \leftrightarrow y$, and as we will see later in the stability analysis same results hold for both the signs. Moreover, we would like to stress here that the solutions which we have obtained are actually the future asymptotic states, i.e., to which spacetime "prefers" to settle down. This makes this analysis very powerful since not only we recover in a very simple fashion a whole class of scale invariant solutions but also, due to "universality", these solutions actually are the preferred asymptotic states.

We will consider now the linear stability analysis. Since these solutions emerge as fixed-points of the RG map, the stability of these solutions is the stability of these fix-points. Let us define

$$
\begin{aligned}
\phi & =\phi^{*}(1+\delta \phi), \\
\psi & =\psi^{*}(1+\delta \psi),
\end{aligned}
$$

where $\delta \phi \ll 1$ and $\delta \psi \ll 1$. The above form of perturbations is chosen to facilitate the analysis since both unperturbed functions $\phi^{*}$ and $\psi^{*}$ can diverge at $z= \pm 1$ and $z= \pm \infty$.

The perturbation equations takes the form

$$
\begin{aligned}
\frac{d \delta \phi}{d \tau} & =\left(\frac{\phi^{*}}{\phi^{*^{\prime}}}\right)\left[\left(\alpha+2 z \frac{\phi^{* \prime}}{\phi^{*}}\right) \frac{d \delta \phi}{d z}+\frac{1}{2} \frac{d \delta \psi}{d z}\right], \\
\frac{d \delta \psi}{d \tau} & =-\left(\frac{\phi^{*}}{\phi^{*^{\prime}}}\right)\left[2\left(\alpha^{2}+\Delta+2 \alpha z \frac{\phi^{* \prime}}{\phi^{*}}\right) \frac{d \delta \phi}{d z}+\alpha \frac{d \delta \psi}{d z}\right] .
\end{aligned}
$$

We shall compute normal modes assuming the following form for the perturbations:

$$
\delta \phi=e^{\omega \tau} \rho(z), \quad \delta \psi=e^{\omega \tau} \sigma(z),
$$

where $\omega$ is a constant. The perturbation equations (3.23) can be written as:

$$
\begin{aligned}
\left(\alpha+2 z \frac{\phi^{* \prime}}{\phi^{*}}\right) \rho^{\prime}+\frac{1}{2} \sigma^{\prime} & =\frac{\phi^{* \prime}}{\phi^{*}} \omega \rho, \\
-2\left(\alpha^{2}+\Delta+2 \alpha z \frac{\phi^{* \prime}}{\phi^{*}}\right) \rho^{\prime}-\alpha \sigma^{\prime} & =\frac{\phi^{* \prime}}{\phi^{*}} \omega \sigma .
\end{aligned}
$$

From these equations it is easy to see that:

$$
\omega \sigma=-2 \sqrt{\triangle} \sqrt{z^{2}-1} \rho^{\prime}-2 \alpha \omega \rho .
$$

Taking the derivative of this equation and substituting in (3.25) we get:

$$
\rho^{\prime \prime}+\frac{z}{z^{2}-1}(1-2 \omega) \rho^{\prime}+\frac{\omega^{2}}{z^{2}-1} \rho=0 .
$$

Thus, the problem of solving linear perturbations around the fixed-points has been reduced to finding solutions of the above equation, and (3.26) completes the solution. The general solution of (3.27) is given by:

$$
\rho(z)=\left(z^{2}-1\right)^{\omega / 2+1 / 4}\left(c_{1} P_{-1 / 2}^{\omega+1 / 2}(z)+c_{2} Q_{-1 / 2}^{\omega+1 / 2}(z)\right),
$$

where $P_{\nu}^{\mu}$ and $Q_{\nu}^{\mu}$ are Legendre functions of first and second kind, respectively, and $c_{1}$ and $c_{2}$ are arbitrary constants. From (3.26) the solution for $\sigma$ can be easily obtained:

$$
\begin{aligned}
\sigma(z)= & -2 \sqrt{\triangle}\left(z^{2}-1\right)^{\omega / 2-1 / 4}\left[c_{1}\left(z P_{-1 / 2}^{\omega+1 / 2}(z)-P_{1 / 2}^{\omega+1 / 2}(z)\right)+c_{2}\left(z Q_{-1 / 2}^{\omega+1 / 2}(z)-Q_{1 / 2}^{\omega+1 / 2}(z)\right)\right] \\
& -2 \alpha\left(z^{2}-1\right)^{\omega / 2+1 / 4}\left(c_{1} P_{-1 / 2}^{\omega+1 / 2}(z)+c_{2} Q_{-1 / 2}^{\omega+1 / 2}(z)\right) .
\end{aligned}
$$

We require that the perturbations be regular for all $z$. Since the differential equation (3.27) has four regular singular points at $z= \pm 1, \pm \infty$ we must consider three different regions: $1<z<\infty,-\infty<z<-1$ and $-1<z<1$. The first and the second region are equivalent. Let's first analyze the behavior in the first region. It is easy to see that 
the leading terms of the two linearly independent solutions $\rho_{1}$ and $\rho_{2}$ of (3.27) in the neighborhood of $z=\infty$ are respectively $\rho_{1} \approx z^{\omega}+O\left(z^{\omega-2}\right)$ and $\rho_{2} \approx \rho_{1} \ln z+O\left(z^{\omega-2}\right)$. Both solutions are regular if $\omega \leq 0$. Furthermore, from (3.26),$\sigma$ is also regular provided that $\omega \leq 0$. At $z=1$ the leading terms of the two linearly independent solutions for $\rho$ are: $(z-1)^{\omega+1 / 2}+O\left((z-1)^{\omega+3 / 2}\right)$ and constant $+O((z-1))$. For $\sigma$ the leading terms are: $(z-1)^{\omega}$ and constant $+O((z-1))$. Since $\omega$ is negative the first independent solution must be neglected so that we have regular solutions. For instance, tacking $c_{2}=0$ in (3.28) the solution is regular.

Making the scale transformation given by (3.7)-(3.9) we get the the behavior of the solution near the fixed-point :

$$
\begin{aligned}
& \delta \phi=t^{\omega} \rho(z / t), \\
& \delta \psi=t^{\omega} \sigma(z / t),
\end{aligned}
$$

with $\omega \leq 0$ and $z>t$. Since the solution above is valid in the region $z>t$, taking limit $t \rightarrow \infty$ means $z \rightarrow \infty$ as well. We can therefore distinguish two different situations. First, when we approach infinity in such a way that we encounter the light-cone $z=t$ without crossing it. In this case asymptotically $z / t=1$ and, bearing in mind the behavior of the regular solutions of (3.27) close to the point $z=1$, the above perturbations behave as:

$$
\delta \phi \approx \delta \psi \approx \text { constant } \times t^{\omega}
$$

In the second situation we approach infinity through a path that does not encounter the light-cone. In this case $z / t$ tends to a constant greater than 1, and the perturbations behave in a similar way that that given by the above expressions. So, in both cases the perturbations tend to zero when $t \rightarrow \infty$ which means that the fixed solution outside the light-cone is stable.

Let's perform this analysis in the region $-1<z<1$. The leading terms of the independent solutions of (3.27)close to $z=1$ are those described earlier for the same point changing $z-1$ by $1-z$. When $z=-1$ the behavior is the same changing $z-1$ by $z+1$. From this we can not obtain a definite conclusion about the values of $\omega$ which make the solution regular. To do that is convenient to transform the equation (3.27) to a hypergeometric differential equation with coefficients $a=b=1 / 2$ and $c=1 / 2-\omega$. It is not difficult to see that one of the independent solutions the solution of (3.27) and (3.26) is given by:

$$
\begin{aligned}
\rho(z)= & c_{1}(1+z)^{\omega+1 / 2} F\left(\frac{1}{2}, \frac{1}{2} ; \frac{1}{2}-\omega ; \frac{1-z}{2}\right) \\
\sigma(z)= & -2 c_{1} \frac{\sqrt{|\triangle|}}{\omega} \sqrt{1-z^{2}}(1+z)^{\omega-1 / 2}\left[\left(\omega+\frac{1}{2}\right) F\left(\frac{1}{2}, \frac{1}{2} ; \frac{1}{2}-\omega ; \frac{1-z}{2}\right)-\frac{1}{4-8 \omega}(1+z) F\left(\frac{3}{2}, \frac{3}{2} ; \frac{3}{2}-\omega ; \frac{1-z}{2}\right)\right] \\
& -2 \alpha c_{1}(1+z)^{\omega+1 / 2} F\left(\frac{1}{2}, \frac{1}{2} ; \frac{1}{2}-\omega ; \frac{1-z}{2}\right) .
\end{aligned}
$$

The form of the second independent solution depends on the values of $\omega$. In any case, the above solution is regular when $\omega$ is positive, except when $\omega=n+1 / 2$ ( $n$ a positive integer $)$. Therefore, with $|z|<t$, the modes with $\omega$ positive dominate and the solution in the interior of the light-cone is not stable.

\section{DISCUSSION}

In this paper we have shown how the RG method can be used to obtain the asymptotic regime of cosmological solutions. We have first illustrated the method applying it to homogenous cosmological models with a scalar field. These models have been extensively analyzed using, mainly, the so called "expansion normalized variables"; which are a set of dimensionless variables [ $[3$. In those works the role of self-similar solutions in describing the asymptotic regime has been stressed. Since a fixed-point of the RG transformation is a scale-invariant solution, it is reasonable to think that the RG method will naturally render the same results (and we have manifestly shown this here, section III) as those obtained using the "expansion normalized variables". It is worthy to stress also that those normalized variables are well adapted to the dimensional and similarity analysis [18]. These results bring the asymptotic behavior of the homogeneous cosmological models in a new perspective. Moreover, its simplicity as well as systematic approach show that it is a practical idea to implement RG for studying asymptotic behavior of spacetimes in General Relativity.

We have also applied the RG method to diagonal, vacuum inhomogeneous $G_{2}$ metric. In this case, the system reduces to a set of coupled partial differential equations whose analysis using the RG method is not difficult. The fixed-point is an exact solution depending on two parameters. This solution belongs to the class of soliton solutions. Soliton solutions are intended as those solutions which can be obtained by the inverse scattering transformation from a known one 20]. In particular, the found fixed-point is a soliton solution with real poles with origin at $z=0$, whose 
"seed" metric is the Kasner metric (soliton origin marks the origin of the light-cone $z^{2}=t^{2}$ ). Since the metric (3.4) is invariant under a $z$ translation the fact that the origin is at $z=0$ is not important. Moreover, a more general class of soliton solutions is that corresponding to a sum of solitons each with a different origin. Since the RG method gives the long-time behavior of the solution, the difference in the origins tend to zero as $t$ tends to infinity leaving only one origin. It is interesting to note that, generically, the solution doesn't homogenize, let alone isotropize, for the final state is inhomogeneous. There is, however, a few particular cases for which the metric tends to a homogeneous solution: when $\triangle=0$ the solution is the Bianchi type I Kasner metric, and for a particular value of the parameters $\alpha$ and $\triangle$ the solution is Ellis and McCallum family of vacuum Bianchi models [25]. Finally, we would like to note that the fixed-points recovered in the RG technique gives all the scale-invariant exact solutions of vacuum $G_{2}$ cosmologies.

Now we can understand the relation of the anomalous dimensions $\alpha$ and $\beta$ with initial conditions: exponent $\alpha$ gives the Kasner parameter of the "seed" metric to generate the soliton solution by means of inverse scattering technique. The parameter $\triangle$ (which fixes $\beta$ ) determines the number of solitons in our solution. So, the two "initial conditions" used in the inverse scattering technique, number of solitons and the Kasner seed metric, fixes the anomalous dimension of system comprising of equations (3.5) and (3.6).

The RG method allows us to study the linear perturbations around the fixed-points as well. We have shown that the solution out-side the light-cone, which corresponds to the soliton solution with real poles, is stable against bounded perturbations, contrary to the solution inside the light-cone (cosoliton solution) which we find to be unstable. Let's note that although the stability analysis doesn't have any apparent dependence on the $\triangle$ parameter one should be very cautious to extend these results to the case $\Delta=0$. This case corresponds to the Kasner solution and this is the only case for which the fixed-point results in $\phi^{* \prime}=\psi^{* \prime}=0$, that makes the system of equations for the perturbations singular.

There remain a few open questions that we hope to deal-with in future works. First is related with the role of soliton solutions with complex poles. These can be obtained as a complex translation along the $z$ axis of the soliton solution with real poles and they are regular in all the spacetime, even on the light-cones. Since this complexification doesn't alter the structure of the field equations it would be worthy to investigate whether the complex pole soliton solutions can represent an asymptotic state of the inhomogeneous metrics. Second, it would be interesting to extend the analysis performed in this paper to general inhomogeneous non-vacuum metrics. Solutions with a scalar field are of particular interest in order to investigate issues like isotropization, scaling solutions, etc.. Finally, this technique can be used to analyze long time behavior of spacetimes with more that four dimensions, for example, brane-world scenarios.

\section{acknowledgements}

We thank Takahiro Tanaka, Oriol Pujolas, M. Valle and J. A. Oteo for discussions. J.I. acknowledges financial support under CICYT grant BFM 2000-0018.

[1] P. J. Adams, R. W. Hellings, R. L. Zimmerman, H. Farhoosh, D. I. Levine and S. Zeldich, Ap. J. 253 (1982) 1; P. J. Adams, R. W. Hellings and R. L. Zimmerman, Ap. J. 288 (1985) 14.

[2] A. Krasinski, Inhomogeneous Cosmological Models, (Cambridge University Press, 1996).

[3] See, chapter 12 in Dynamical Systems in Cosmology, Ed. J. Wainwright and G. F. R. Ellis (Cambridge University Press, 1997), and references therein.

[4] J. Ibáñez and I. Olasagasti, J. Math. Phys. 37 (1996) 6238.

[5] J. Carot and M. M. Collinge, Class. Quant. Grav. 18 (2001) 5441.

[6] B. J. Carr and A. A. Coley, Class. Quant. Grav. 16 (1999) R31.

[7] J. Bricmont, A. Kupiainen and G. Lin, Commun. Pure and Appl. Math. XLVII (1994) 893.

[8] J. Bricmont and A. Kupiainen, Renormalizing Partial Differential Equations, in Constructive Physics, (ed. V. Rivasseau, Springer, 1995) chao-dyn/9411015.

[9] L. Y. Chen, N. Goldenfeld and Y. Oono, Phys. Rev. Lett. 73 (1994) 1311.

[10] L. Y. Chen, N. Goldenfeld and Y. Oono, Phys. Rev. E, 54 (1996) 376.

[11] O. Iguchi, A. Hosoya and T. Koike, Phys. Rev. D 57 (1998) 3340.

[12] T. Koike, T. Hara and S. Adachi, Phys. Rev. Lett. 74 (1995) 5170.

[13] Y. Sota, T. Kobayashi, K. Maeda, T. Kurokawa, M. Morikawa and A. Nakamichi, gr-qc/9801083

[14] J. A. Belinchón, T. Harko and M. K. Mak, Class. Quant. Grav. 19 (2002) 3003.

[15] A. Bonnano and M. Reuter, Phys. Lett. B 527 (2002) 9; M. Reuter and H. Weyer, Phys. Rev. D 69 (2004) 104022 ; A. Bonnano and M. Reuter, Int. J. Mod. Phys. D 13 (2004) 107.

[16] I. L. Shapiro, J. Sola, C. Espana-Bonet and P. Ruiz-Lapuente Phys. Lett. B 574 (2003) 149. 
[17] N. Goldenfeld, O. Martin and Y. Oono, J. Sci. Comput. 4 (1989) 355.

[18] G. I. Barenblatt, Similarity, Self-Similarity and Intermediate Asymptotics, (Consultants Bureau, New York, 1989).

[19] A. A. Coley, J. Ibáñez and R. J. van den Hoogen, J. Math. Phys. 38 (1997) 5256.

[20] E. Verdaguer, Phys. Rep. 229 (1993) 1.

[21] M. Carmeli, Ch. Charach and S. Malin, Phys. Rep. 76 (1981) 79.

[22] V. A. Belinskii, E. M. Lifshitz and I. M. Khalatnikov, Sov. Phys. Uspechi 13 (1971) 745.

[23] N. Goldenfeld, Lectures on Phase Transitions and the Renormalization Group, Frontiers in Physics Series 85 (AddisonWesley publishing company, 1985).

[24] V. Belinski and E. Verdaguer, Gravitational Solitons, (Cambridge University Press, 2001).

[25] G. F. R. Ellis and M. A. H. MacCallum, Commun. Math. Phys. 12 (1969) 108. 